\title{
Modeling of a natural lipstick formulation using an artificial neural network
}

\begin{abstract}
An artificial neural network (ANN) was applied in conjunction with experimental data from a mixture of experimental designs to predict the melting point of a lipstick formulation. The experimental data were utilized for training and testing the suggested model. By using the ANN performance results, the optimum parameters were found to be pitaya seed oil $25 \% \mathrm{w} / \mathrm{w}$, virgin coconut oil $37 \% \mathrm{w} / \mathrm{w}$, beeswax $17 \% \mathrm{w} / \mathrm{w}$, candelilla wax $2 \% \mathrm{w} / \mathrm{w}$, and carnauba wax $2 \% \mathrm{w} / \mathrm{w}$. The relative standard error under these parameters was only $0.8772 \%$. It was found that batch back-propagation (BBP) gave the optimal algorithm and topology with a configuration of five inputs, two hidden nodes and one output node; the most important parameter was the carnauba wax content with a relative importance of $24.5 \%$.
\end{abstract}

